\title{
Acute pancreatitis associated with hemorrhagic fever with renal syndrome: a cohort study of 346 patients
}

\author{
Qinyue Guo ${ }^{1}$, Jing $X u^{2}$, Qindong Shi ${ }^{1}$ and Bin $\mathrm{Du}^{3^{*}} \mathrm{D}$
}

\begin{abstract}
Background: To assess the prevalence, risk factors, clinical characteristics, and outcomes of acute pancreatitis (AP) in patients with hemorrhagic fever with renal syndrome (HFRS).

Methods: All patients diagnosed with HFRS admitted to the First Affiliated Hospital of Xi'an Jiaotong University from January 2013 to July 2020 were enrolled. Patients with and without AP were compared by two risk stratification models: (1) a multivariate regression analysis using propensity score to adjust for confounding and (2) a propensity-matched nested case-control study.

Results: A total of 346 patients were enrolled in the cohort study, 29 of whom (8.4\%) were diagnosed as AP. There was no significant difference between patients with and without AP with regards to common risk factors and presenting signs/symptoms other than gastrointestinal symptoms $(p<0.01)$. The patients with AP had a significantly higher 90 -day mortality rate $(24.1 \%$ vs. $3.5 \%, \mathrm{OR} 8.9,95 \% \mathrm{Cl} 1.3$ to $103.4, p=0.045)$, and significantly shorter duration of therapy free-days to 28 day such as RRT and mechanical ventilation free days ( $p<0.05$, respectively).

Conclusions: Our study indicated that AP was independently associated with higher mortality in HFRS patients. While considering the difficulty of early recognition of AP among HFRS patients with similar signs and/or symptoms, further laboratory and imaging studies might help identify these patients at risk of poor clinical prognosis.
\end{abstract}

Keywords: Hemorrhagic fever with renal syndrome, Acute pancreatitis, Hantavirus, Propensity score match

\section{Background}

Hemorrhagic fever with renal syndrome (HFRS) is an acute infectious disease caused by Hantavirus mainly through inhalation of aerosols or dust particles contaminated by virus-containing rodent excreta [1]. Despite the widespread administration of vaccine, HFRS remains endemic in Asia and Europe, while increased incidence

\footnotetext{
* Correspondence: dubin98@gmail.com

${ }^{3}$ State Key Laboratory of Complex, Severe and Rare Diseases, Medical Intensive Care Unit, Peking Union Medical College Hospital, Peking Union Medical College and Chinese Academy of Medical Sciences, 1 Shuai Fu Yuan, Beijing 100730, China

Full list of author information is available at the end of the article
}

have been reported in China [2,3], and selected areas of Europe, such as Germany [4].

Clinical characteristics of HFRS include hemorrhagic manifestations, renal failure, or even multiple organ dysfunction [5] Moreover, due to the lack of definitive therapy, treatment of HFRS remains supportive [6], with a mortality rate about $12 \%$ [7].

Acute pancreatitis (AP) is a rare but life-threatening complication of Hantavirus infection, with significant morbidity and mortality [8]. However, early detection of AP remains challenging, due to the high prevalence of abdominal pain (up to 64.6\%) in HFRS patients even without AP [9]. 
Unfortunately, there have been very few reports of AP in patients with HFRS. We performed this retrospective cohort study to investigate the risk factors, clinical manifestations, laboratory abnormalities, and clinical outcomes of AP among patients with HFRS in an endemic area in China [10-12].

\section{Methods}

\section{Study population}

We retrospectively reviewed the medical records of all adult patients with confirmed HFRS who were admitted to the First Affiliated Hospital of Xi'an Jiaotong University from January 2013 to July 2020. The diagnosis was based on compatible clinical manifestation and laboratory detection of serum IgG antibodies using indirect immunofluorescent antibody test or serum IgM antibodies by enzyme-linked immunosorbent assay (ELISA) method. This study was approved by the institutional review board. Informed consent was waived due to the retrospective nature of the study.

\section{Study design}

The first part of this study was a retrospective cohort study that included all patients with confirmed HFRS.
The outcome of patients with AP was compared with that of patients without AP. The second part of the study was a matched (1:1) case-control study. For the purpose of this study, the patients with AP are designated as case subjects, and those without AP as control subjects. A schematic flow chart is shown in Fig. 1.

\section{Definitions}

AP was diagnosed and graded according to the Atlanta criteria [13]. The detail was that the presence of two of the following three criteria: acute onset of persistent, severe, epigastric pain often radiating to the back, elevation in serum lipase or amylase to three times or greater than the upper limit of normal, and characteristic findings of acute pancreatitis on imaging (contrast-enhanced computed tomography [CT], magnetic resonance imaging [MRI], or transabdominal ultrasonography). Shock was defined as hypotension requiring administration of vasopressors to maintain mean arterial pressure (MAP) > $65 \mathrm{mmHg}$ [14]. Multidrug resistant organisms (MDROs) were microorganisms that were resistant to one or more therapeutic classes of antimicrobial agents [15]. Cardiovascular diseases included coronary heart disease, hypertension, and valvular heart disease, chronic liver diseases

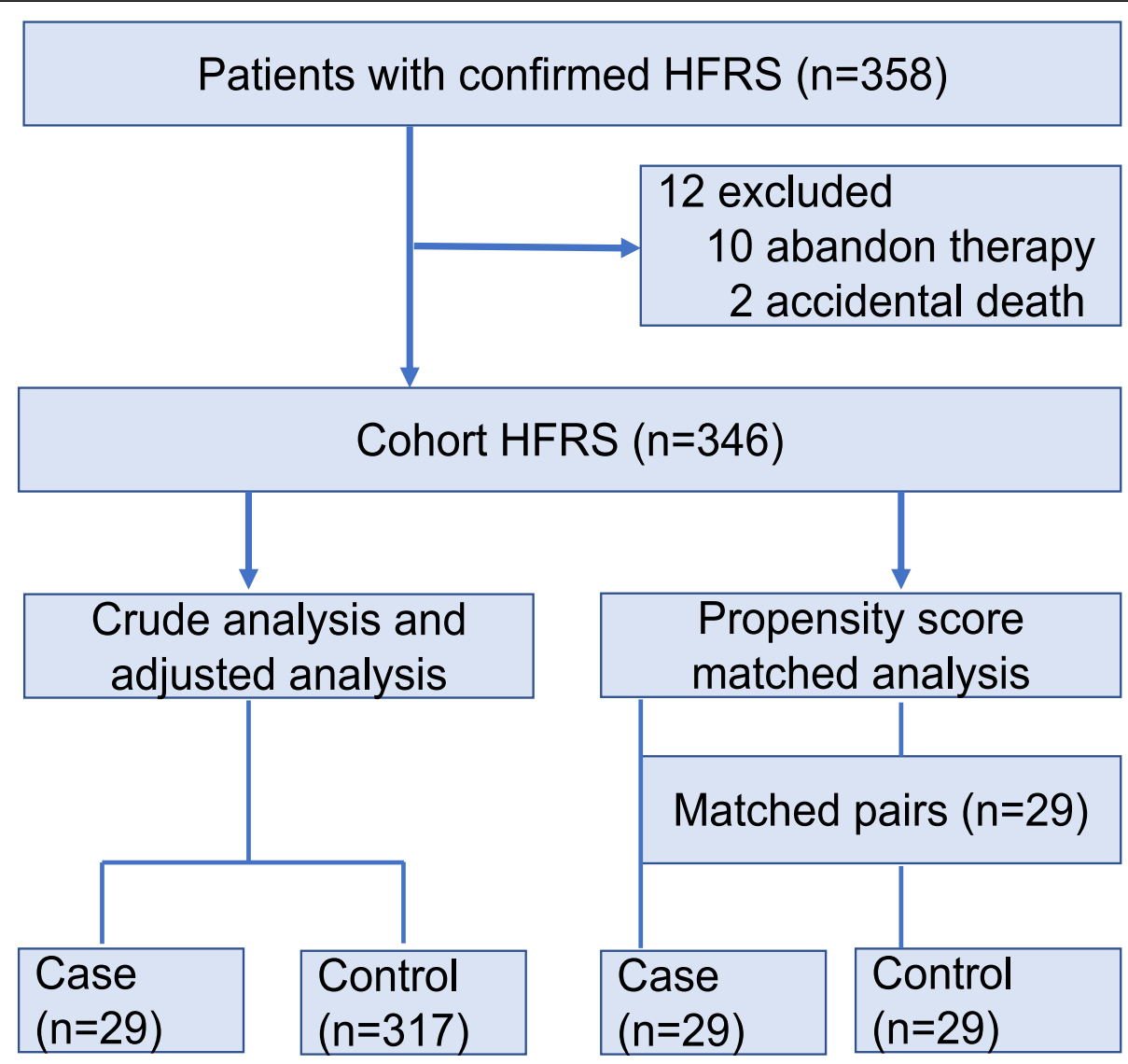

Fig. 1 Schematic flow chart for a cohort study and matched case-control study 
included chronic hepatitis and cirrhosis, whereas chronic respiratory disease referred to chronic obstructive pulmonary disease, bronchitis, and interstitial lung disease.

\section{Data collection}

Data collected included: (1) demographics (sex, age, and living habits); (2) comorbidities, such as biliary tract disease (cholecystitis, cholangitis, gallstones), diabetes, respiratory disease, tumor; (3) the course of HFRS: date of onset, symptoms at presentation, date of hospital admission, and complications (such as shock and MDROs infection); (4) laboratory findings on hospital admission: complete blood count, blood chemistry, and inflammatory biomarkers (C-reactive protein [CRP], procalcitonin $[\mathrm{PCT}]),(5)$ treatment: intensive care unit (ICU) admission, mechanical ventilation, renal replacement therapy (RRT); and (6) outcome measures: 90-day all-cause mortality rate, and hospital-free days at day 28 .

\section{Statistical analysis}

Categorical variables were examined by Fisher exact test or chi-square test, as appropriate, while continuous variables were compared by student $\mathrm{t}$ test or Mann-Whitney $\mathrm{U}$ test. All tests of significance were two-tailed and a $P$ value of 0.05 was considered significant.

In order to account for potential confounding factors in this observational study, we developed a propensity score, using multivariate logistic regression analysis without regards to outcomes [16, 17], to adjust for the differences in baseline characteristics between HFRS patients with and without AP. All prespecified covariates (age, sex, smoker, alcoholics, whether there are comorbidities, days from symptom onset to hospitalization, whether there are gastrointestinal symptoms), as outlined above, were included in the final prediction model for AP among HFRS patients, by means of stepwise backward elimination with $p$ value $<0.1$. Model discrimination was assessed by area under the receiver operating characteristics curve (AUROC).

The effect of AP on 90-day mortality, as well as other clinical outcomes (i.e. ICU admission, RRT, mechanical ventilation, MDRO infection), was analyzed by stepwise backward logistic regression model by including any covariate with $p$ value $<0.10$ in univariate analysis. Moreover, individual propensity score was also included in the model as a covariable to calculate the adjusted odds ratios (aOR) and 95\% confidence intervals (CIs). In addition, we performed a nested case-control study (1:1 match) by matching case and control subjects using calipers of width equal to 0.2 of the standard deviation of the logit of the propensity score $[18,19]$. A nearestneighbor matching algorithm was employed to form pairs of case and control subjects, once a match was made, previous matches were not reconsidered before making the next match. Survival curves for case and control subjects were analyzed by the Kaplan-Meier method and compared by log-rank test.

Results were analyzed with SPSS version $22.0 \mathrm{~K}$ for Windows (SPSS Inc., Chicago, IL, USA) and STATA SE 14 (StataCorp LP, College Station, TX, USA).

\section{Results}

\section{Patient enrolment and clinical characteristics}

During the study period, 358 patients with the diagnosis of HFRS were admitted to the First Affiliated Hospital of Xi'an Jiaotong University. After exclusion of 12 patients due to withholding or withdrawal of life-sustaining therapies or accidental death, 346 patients (mean age 46.1, 255 men [73.7\%]) were included in the final analysis (Fig. 1).

One hundred and forty-seven patients had comorbidities, such as cardiovascular disease $(n=45,13.0 \%)$, diabetes $(n=25,7.2 \%)$, chronic liver disease $(n=24,6.9 \%)$, biliary tract disease $(n=11,3.2 \%)$, and others. The most common presenting signs and symptoms included fever $(n=302,87.3 \%)$, back/flank pain $(n=213,61.6 \%)$, gastrointestinal symptoms $(n=118,34.1 \%)$, and shock $(n=33$, 9.5\%). Of 118 patients with presenting gastrointestinal symptoms, 45 had abdominal pain, 13 had nausea/ vomiting, 48 had diarrhea, and 12 had abdominal distention. RRT and mechanical ventilation were used in 86 (24.9\%) and 17 (4.9\%) patients, respectively. A total of 25 patients $(7.2 \%)$ were admitted to ICU, and 14 patients (4.0\%) died at 90 days.

\section{Patients with acute pancreatitis and development of propensity score}

A total of 29 patients (8.4\%) were diagnosed as AP, including 19 patients having AP on hospital admission, and 10 patients developing AP during hospitalization. Compared with HFRS patients without AP, patients with AP were more likely to be smokers and alcoholics (Table 1). There was no significant difference between patients with and without AP with regards to common risk factors (biliary tract disease, diabetes and other underlying diseases) and presenting signs/symptoms (fever, back/flank pain, and shock) other than gastrointestinal symptoms $(p<0.01)$ (Table 1$)$.

On hospital admission, compared with patients without AP, patients with AP were characterized by more pronounced inflammation (as suggested by higher white cell count, higher CRP), lower platelet count, hypocalcemia, and abnormal liver functions (as suggested by higher liver enzymes, higher bilirubin, and lower albumin) (Table 2). In addition, patients in both groups suffered from mild to moderate renal dysfunction, as indicated by elevated serum creatinine and BUN levels. Interestingly, there was no significant difference between 
Table 1 Baseline characteristics and clinical outcome of hemorrhagic fever renal syndrome patients with and without acute pancreatitis

\begin{tabular}{|c|c|c|c|}
\hline & Case $(n=29)$ & Control $(n=317)$ & $P$ value \\
\hline Age, year (mean $\pm S D)$ & $44.4 \pm 16.4$ & $46.2 \pm 17.3$ & 0.63 \\
\hline Male sex & $19(65.5 \%)$ & $236(74.4 \%)$ & 0.32 \\
\hline Smoker & $11(37.9 \%)$ & $132(41.6 \%)$ & 0.02 \\
\hline Alcoholics & $9(31.0 \%)$ & $40(12.6 \%)$ & 0.03 \\
\hline \multicolumn{4}{|l|}{ Comorbidities } \\
\hline No comorbidities & $14(48.3 \%)$ & $185(58.4 \%)$ & 0.33 \\
\hline Cardiovascular disease & $6(20.7 \%)$ & 39 (12.3\%) & 0.24 \\
\hline Diabetes & $2(6.9 \%)$ & $23(7.3 \%)$ & $>0.99$ \\
\hline Chronic liver disease & $2(6.9 \%)$ & $22(6.9 \%)$ & $>0.99$ \\
\hline Biliary tract disease & $3(10.3 \%)$ & $8(2.5 \%)$ & 0.06 \\
\hline Chronic respiratory disease & $2(6.9 \%)$ & $9(2.8 \%)$ & 0.23 \\
\hline Pregnancy & $1(3.5 \%)$ & $4(1.3 \%)$ & 0.36 \\
\hline Chronic renal disease & $0(0)$ & $4(1.3 \%)$ & $>0.99$ \\
\hline Solid tumor & $0(0)$ & $2(0.6 \%)$ & $>0.99$ \\
\hline Others & $2(6.9 \%)$ & 37 (11.7\%) & 0.76 \\
\hline Days from symptom onset to hospitalization, $\mathrm{d}($ mean $\pm \mathrm{SD})$ & $4.6 \pm 2.6$ & $6.4 \pm 5.4$ & 0.02 \\
\hline \multicolumn{4}{|l|}{ Presenting symptoms } \\
\hline Fever & $24(82.8 \%)$ & $278(87.7 \%)$ & 0.39 \\
\hline Back/Flank pain & $14(48.3 \%)$ & $199(62.8 \%)$ & 0.16 \\
\hline Gastrointestinal symptoms & $20(69.0 \%)$ & $98(30.9 \%)$ & $<0.01$ \\
\hline Nausea/vomiting & $2(6.9 \%)$ & $11(3.5 \%)$ & 0.30 \\
\hline Abdominal pain & $8(27.6 \%)$ & $37(11.7 \%)$ & 0.04 \\
\hline Diarrhea & $7(24.1 \%)$ & $41(12.9 \%)$ & 0.09 \\
\hline Abdominal distention & $3(10.3 \%)$ & $9(2.8 \%)$ & 0.06 \\
\hline Shock & $5(17.2 \%)$ & $28(8.8 \%)$ & 0.18 \\
\hline \multicolumn{4}{|l|}{ Treatment } \\
\hline Antibiotic therapy (mean \pm SD) & $1.7 \pm 1.2$ & $0.7 \pm 0.8$ & 0.01 \\
\hline ICU admission & $13(44.8 \%)$ & $12(3.8 \%)$ & $<0.01$ \\
\hline ICU-free days at day $28, \mathrm{~d}$ (mean $\pm \mathrm{SD})$ & $19.1 \pm 10.2$ & $27.1 \pm 4.8$ & $<0.01$ \\
\hline RRT & $17(58.6 \%)$ & $69(21.8 \%)$ & $<0.01$ \\
\hline RRT-free days at day $28, d$ (mean \pm SD) & $18.6 \pm 11.4$ & $25.2 \pm 6.5$ & $<0.01$ \\
\hline Mechanical ventilation & $9(31.0 \%)$ & $8(2.5 \%)$ & $<0.01$ \\
\hline Ventilator-free days at day $28, \mathrm{~d}($ mean $\pm \mathrm{SD})$ & $21.3 \pm 11.5$ & $27.4 \pm 4.1$ & $<0.01$ \\
\hline \multicolumn{4}{|l|}{ Clinical outcome } \\
\hline 90-day mortality & $7(24.1 \%)$ & $7(2.2 \%)$ & $<0.01$ \\
\hline Hospital-free days at day $28, \mathrm{~d}$ (mean $\pm \mathrm{SD}$ ) & $8.6 \pm 8.4$ & $16.4 \pm 6.6$ & 0.005 \\
\hline
\end{tabular}

ICU Intensive care unit, RRT Renal replacement therapy

Data are presented as the number (percentage) of patients unless indicated otherwise

the two groups with regards to serum lipase and serum/ urine amylase levels on hospital admission (Table 2).

Based on above data, we developed a propensity score using a multivariate logistic regression model with an area under the receiver operating characteristics curve of 0.71 (95\%CI 0.62 to 0.81 ) (Supplementary material
Figure), indicating good discrimination of HFRS patients developing AP.

\section{Acute pancreatitis as risk factor for clinical outcome}

Compared with patients without AP, patients with AP were more severe, as indicated by more antibiotic 
Table 2 Laboratory results on hospital admission in hemorrhagic fever renal syndrome patients with and without acute pancreatitis

\begin{tabular}{|c|c|c|c|}
\hline & Case $(n=29)$ & Control $(n=317)$ & $P$ value \\
\hline$\overline{W C C}\left(\times 10^{9} / \mathrm{L}\right)$ & $20.0 \pm 15.2$ & $11.9 \pm 9.1$ & 0.01 \\
\hline Platelet $\left(\times 10^{9} / \mathrm{L}\right)$ & $55.5 \pm 84.0$ & $110.3 \pm 98.1$ & 0.01 \\
\hline Neutrophil (\%) & $76.9 \pm 27.2$ & $63.9 \pm 19.2$ & 0.01 \\
\hline C-reactive protein (mg/L) & $45.8 \pm 31.4$ & $30.9 \pm 19.5$ & 0.02 \\
\hline Procalcitonin (ng/ml) & $12.9 \pm 37.0$ & $4.8 \pm 11.8$ & 0.20 \\
\hline Albumin (g/L) & $29.5 \pm 5.6$ & $34.8 \pm 7.0$ & 0.01 \\
\hline Total bilirubin $(\mu \mathrm{mol} / \mathrm{L})$ & $15.6 \pm 6.9$ & $11.2 \pm 5.3$ & 0.01 \\
\hline AST $(U / L)$ & $194.8 \pm 261.6$ & $67.2 \pm 100.8$ & 0.01 \\
\hline $\operatorname{ALT}(U / L)$ & $83.0 \pm 84.6$ & $42.9 \pm 24.5$ & 0.02 \\
\hline $\operatorname{ALP}(U / L)$ & $78.67 \pm 53.9$ & $99.7 \pm 32.8$ & 0.75 \\
\hline $\mathrm{LDH}(\mathrm{U} / \mathrm{L})$ & $1056 \pm 1150$ & $547.9 \pm 280.8$ & 0.04 \\
\hline Triglyceride $(\mathrm{mmol} / \mathrm{L})$ & $3.21 \pm 1.91$ & $9.89 \pm 75.2$ & 0.93 \\
\hline Low density lipoprotein (mmol/L) & $1.44 \pm 1.05$ & $1.8 \pm 0.6$ & 0.29 \\
\hline High-density lipoprotein (mmol/L) & $0.6 \pm 0.2$ & $0.9 \pm 0.8$ & 0.14 \\
\hline Serum creatinine $(\mu \mathrm{mol} / \mathrm{L})$ & $292.2 \pm 260.6$ & $217.5 \pm 205.8$ & 0.14 \\
\hline $\mathrm{BUN}(\mathrm{mmol} / \mathrm{L})$ & $15.1 \pm 10.7$ & $11.7 \pm 9.0$ & 0.12 \\
\hline Serum calcium (mmol/L) & $1.8 \pm 0.2$ & $2.0 \pm 0.2$ & 0.01 \\
\hline Serum amylase (U/L) & $257.9 \pm 232.4$ & $170.7 \pm 97.8$ & 0.39 \\
\hline Urine amylase (U/L) & $200.2 \pm 194.1$ & $133 \pm 26.1$ & 0.65 \\
\hline Serum lipase (U/L) & $1261.0 \pm 1068.2$ & $988.2 \pm 899.1$ & 0.39 \\
\hline
\end{tabular}

ALT Alanine aminotransferase, ALP Alkaline phosphatase, AST Aspartate aminotransferase, BUN Blood urea nitrogen, LDH Lactate dehydrogenase, WCC White blood cell count

Data are presented as the mean \pm SD

combination therapy, more life-sustaining therapies (i.e. mechanical ventilation, RRT), more ICU admissions (44.8\% vs. $3.8 \%$, OR $20.7,95 \%$ CI 8.4 to $53.5, p<0.01)$, and shorter hospital-free days, as well as higher 90-day mortality rate $(24.1 \%$ vs. $2.2 \%$, OR $14.1,95 \%$ CI 4.8 to 40.4, $p<0.01$ ) (Tables 1 and 3).

In multivariate regression analysis adjusted for potential confounders (smoker, alcoholics, whether there are comorbidities, days from symptom onset to hospitalization, whether there are gastrointestinal symptoms) with propensity score, AP was associated with increased 90-day mortality rate (adjusted odds ratio $[\mathrm{aOR}], 17.6,95 \% \mathrm{CI}, 4.3$ to $73.3, p<0.01)$. In addition, AP was an independent risk factor of ICU admission (aOR, 22.8; 95\% CI, 7.8 to $66.4, p<0.01$ ), mechanical ventilation (aOR, 32.5; 95\% CI, 7.9 to 134.4, $p<0.01$ ), and RRT (aOR, 4.3; 95\% CI, 1.9 to 9.6, $p<0.01)$ (Table 3).

Table 3 Analysis of acute pancreatitis associated with 90-day mortality rate and supportive treatment in 346 patients with hemorrhagic fever renal syndrome

\begin{tabular}{|c|c|c|c|c|c|c|}
\hline & Crude & & Adjusted & & Propensity-matcl & \\
\hline & OR $(95 \% \mathrm{Cl})$ & $P$ Value & OR $(95 \% \mathrm{Cl})$ & $P$ Value & OR (95\% Cl) & $P$ Value \\
\hline 90-day mortality & $14.1(4.8-40.4)$ & $<0.01$ & $17.6^{\mathrm{a}}(4.3-73.3)$ & $<0.01$ & $8.9(1.3-103.4)$ & 0.045 \\
\hline ICU admissions & $20.7(8.4-53.5)$ & $<0.01$ & $22.8^{\mathrm{a}}(7.8-66.4)$ & $<0.01$ & $22.8(3.3-249.6)$ & $<0.01$ \\
\hline RRT & $5.1(2.3-11.3)$ & $<0.01$ & $4.3^{b}(1.9-9.6)$ & $<0.01$ & $3.2(1.1-8.7)$ & 0.06 \\
\hline Mechanical ventilation & $17.4(6.1-52.4)$ & $<0.01$ & $32.5^{\mathrm{a}}(7.9-134.4)$ & $<0.01$ & $12.6(1.64-142.4)$ & 0.01 \\
\hline MDRO infection & $7.8(1.3-38.8)$ & 0.06 & $5.02^{\mathrm{C}}(0.5-46.9)$ & 0.16 & $2.07(0.2-31.0)$ & $>0.99$ \\
\hline
\end{tabular}

CI Confidence interval, OR Odds ratio, ICU Intensive care unit, RRT Renal replacement therapy, MDRO Multiple drug resistant organism

Adjusted for variables ( ${ }^{a}$ alcoholics; ${ }^{b}$ alcoholics, days from symptom onset to hospitalization; ${ }^{C}$ alcoholics, days from symptom onset to hospitalization, gastrointestinal symptoms) associated with 90-day mortality, treated in ICU, treated with CRRT, treated with mechanical ventilation, infected with MDRO and the propensity score of each patient's likelihood of being diagnosed with acute pancreatitis

${ }^{d}$ Of 346 patients, 29 pairs were matched 


\section{Matched case-control study}

In propensity score-matched case-control study, 29 HFRS patients with AP (case subjects) were successfully matched with 29 HFRS patients without AP (control subjects) (Supplementary material Table 1). Case subjects were more likely to receive mechanical ventilation (OR, 12.6; 95\% CI, 1.64 to $142.4, p=0.01$ ), ICU admission (OR, 22.8; 95\% CI, 3.3 to 249.6, $p<0.01$ ), but not RRT (OR, 3.2; 95\% CI, 1.1 to 8.7, $p=0.06$ ) (Supplementary material Table 2). Compared with control subjects, case subjects had a significantly higher 90-day mortality rate $(24.1 \%$ vs. $3.5 \%$, OR $8.9,95 \%$ CI 1.3 to $103.4, p=$ 0.045) (Table 3, and Fig. 2), and shorter RRT-free days and ventilator-free days (both $p<0.05$ ) (Supplementary material Table 2).

\section{Discussion}

Diverse clinical manifestations had been reported in HFRS patients, ranging from mild and acute influenzalike illness to more severe shock syndrome. Apart from acute renal insufficiency, up to one-third of HFRS patients exhibited extrarenal organ involvement, with pancreatobiliary diseases as the most common manifestation, including acalculous cholecystitis, pancreatitis, and cholangitis [9]. Hullinghorst and Steer reported pathological evidence of pancreatitis in one-third of HFRS autopsies during the Korean conflict [20]. The reported prevalence of AP among HFRS patients was highly variable in observational studies, ranging from 2.8 to $78 \%$, with a pooled prevalence of $6.8 \%(36 / 529)$ [9, 21-25]. We found that $8.4 \%$ of HFRS patients in our cohort developed AP. The observed difference in prevalence of AP among HFRS patients might be related, at least in part, to the causative viruses [9], geographic region [24], male-to-female ratio [8], prevalence of risk factors (such as alcohol misuse and history of gallstones), and time course during disease progression. For example, the highest prevalence of AP was reported by Bui-Mansfield and colleagues in a group of 13 male patients with HFRS [22], whereas the lowest prevalence was reported by Zhu and coworkers in 218 HFRS patients (150 males and 68 females) [23].

Another major finding of our study was that AP was an independent risk factor for 90-day mortality in HFRS patients, which had never been studied in the abovementioned observational studies, possibly due to the limited number of AP cases (3 to 12) in the individual study $[8,9,21-25]$. However, pooled results from these studies suggested similar mortality rates $[8.3 \%(3 / 36)$ in HFRS patients with AP vs $4.9 \%$ (24/493) in HFRS patients without AP, $p=0.3618$ ]. In comparison, the 29 cases in our cohort represented the largest number of AP cases among HFRS patients ever reported, which allowed us to investigate the impact of AP on mortality in univariate and multivariate analyses. The significantly higher mortality rate in HFRS patients with AP ( $24.1 \%$ vs. $2.2 \%)$ could be explained by the severity of acute illness, as demonstrated by more pronounced inflammation (higher white cell count and CRP levels), liver dysfunction, more life-sustaining therapies (including mechanical ventilation and RRT), and more ICU admissions (44.8\% vs. $3.8 \%)$.

It is a common belief that early recognition of patients with AP might be very important to improve clinical outcome of this potentially life-threatening condition. However, early diagnosis of AP among HFRS patients might be difficult, as both diseases shared some common clinical signs/symptoms, such as nausea/vomiting and

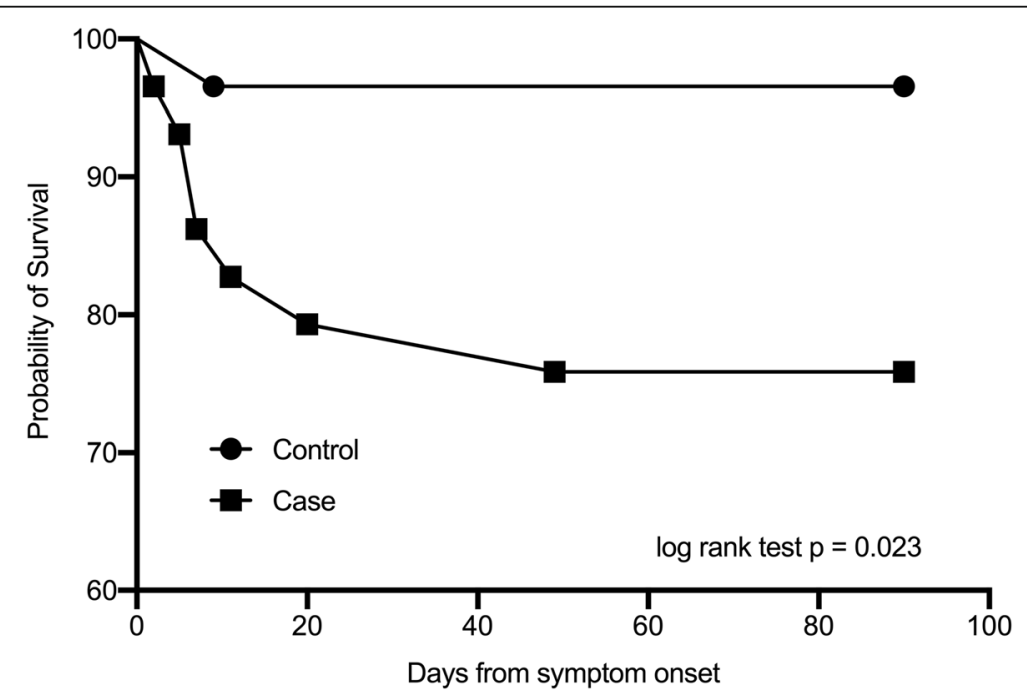

Fig. 2 Kaplan-Meier survival curve of 29 pairs of HFRS patients stratified by the presence of acute pancreatitis. HFRS, hemorrhagic fever with renal syndrome 
abdominal pain. For example, abdominal pain was a presenting symptom in 30 to $90 \%$ of HFRS patients, which might explain the observed high misdiagnosis rate (up to 90\%) [8]. As a result, HFRS patients with abdominal pain should be subject to follow-up laboratory (i.e. pancreatic and/or liver enzymes) and imaging (i.e. abdominal CT scan or ultrasonography) investigations [9, 24], in order to determine the presence and severity of pancreatobiliary complications.

The major strength of our study was the robustness of the study result (i.e. AP as an independent risk factor for mortality), which was supported by univariate analysis, multivariate regression analysis adjusted for propensity score, and propensity score-matched case-control analysis. Our study was also subject to limitations. First, this was a retrospective single-center study, the result of which might not be generalized to other settings and required further validation by prospective multicenter studies. Nonetheless, the number of AP cases as well as HFRS patients in our cohort was significantly higher than that in previous studies. Second, the prevalence of AP might be underestimated, because laboratory (serum amylase or lipase) and abdominal imaging investigations were only performed in selected but not all HFRS patients. Last, we only compared laboratory tests on hospital admission, when only 19 out of 29 patients with AP were diagnosed as such, in whom the baseline laboratory tests at AP onset were not available.

That may explain why there was no difference in serum lipase and serum/urinary amylase of two groups.

\section{Conclusions}

In conclusion, our study indicated that AP was independently associated with higher mortality in HFRS patients. While considering the difficulty of early recognition of AP among HFRS patients with similar signs and/or symptoms, further laboratory and imaging studies might help identify these patients at risk of poor clinical prognosis.

\section{Abbreviations}

AP: Acute pancreatitis; HFRS: Hemorrhagic fever with renal syndrome; ELISA: Enzyme-linked immunosorbent assay; CT: Computed tomography; MRI: Magnetic resonance imaging; MDROs: Multidrug resistant organisms; CRP: C-reactive protein; PCT: Procalcitonin; ICU: Intensive care unit; RRT: Renal replacement therapy; AUROC: Area under the receiver operating characteristics curve; Cl: Confidence interval; OR: Odds ratio; ALT: Alanine aminotransferase; ALP: Alkaline phosphatase; AST: Aspartate aminotransferase; BUN: Blood urea nitrogen; LDH: Lactate dehydrogenase; WCC: White blood cell count

\section{Supplementary Information}

The online version contains supplementary material available at https://doi. org/10.1186/s12879-021-05964-5.

Additional file 1.

\section{Acknowledgements}

Not applicable.

\section{Authors' contributions}

QYG and BD had full access to all of the data in the study and take responsibility for the integrity of the data and the accuracy of the data analysis. Concept and design: QYG and BD. Acquisition, analysis, or interpretation of data: JX and QDS. Drafting of the manuscript: QYG. Critical revision of the manuscript for important intellectual content: BD. Statistical analysis: QYG, BD. Supervision: BD. The authors read and approved the final manuscript.

\section{Funding}

No funding supported.

Availability of data and materials

The datasets analyzed during the current study are available from the corresponding author upon reasonable request.

\section{Declarations}

\section{Ethics approval and consent to participate}

This study was approved by the Xi'an Jiaotong University Institutional Review Board and The First Affiliated Hospital of Xi'an Jiaotong University Clinical Research Review Committee and a waiver of informed consent was granted. Data collected from the study participants was kept anonymous and treated as confidential at all times.

\section{Consent for publication}

Not Applicable.

\section{Competing interests}

All the authors have fulfilled all conditions required for authorship and have approved the submission. None of the authors has any conflicts of interest to disclose.

\section{Author details}

'Department of Critical Care Medicine, The First Affiliated Hospital of Xi'an Jiaotong University, 277 Yanta West Street, Xi'an 710061, Shaanxi, China. ${ }^{2}$ Department of Emergency Medicine, The First Affiliated Hospital of Xi'an Jiaotong University, 277 Yanta West Street, Xi'an 710061, Shaanxi, China. ${ }^{3}$ State Key Laboratory of Complex, Severe and Rare Diseases, Medical Intensive Care Unit, Peking Union Medical College Hospital, Peking Union Medical College and Chinese Academy of Medical Sciences, 1 Shuai Fu Yuan, Beijing 100730, China.

Received: 22 October 2020 Accepted: 8 March 2021

Published online: 17 March 2021

\section{References}

1. Goeijenbier M, Wagenaar J, Goris M, et al. Rodent-borne hemorrhagic fevers: under-recognized, widely spread and preventable-epidemiology, diagnostics and treatment [J]. Crit Rev Microbiol. 2013;39(1):26-42. https:// doi.org/10.3109/1040841X.2012.686481.

2. Arai S, Gu SH, Baek $\sqcup$, et al. Divergent ancestral lineages of newfound hantaviruses harbored by phylogenetically related crocidurine shrew species in Korea []]. Virology. 2012;424(2):99-105. https://doi.org/10.1016/j.virol.2011.11.013.

3. Park Y. Epidemiologic study on changes in occurrence of hemorrhagic fever with renal syndrome in Republic of Korea for 17 years according to age group: 2001-2017[]]. BMC Infect Dis. 2019;19(1):1-8.

4. Reusken C, Heyman P. Factors driving hantavirus emergence in Europe. [J]. Curr Opin Virol. 2013;3(1):92-9. https://doi.org/10.1016/j.coviro.2013.01.002.

5. Tadin A, Turk N, Korva M, et al. Multiple co-infections of rodents with hantaviruses, Leptospira, and Babesia in Croatia[J]. Vector-Borne Zoonotic Dis. 2012;12(5):388-92. https://doi.org/10.1089/vbz.2011.0632.

6. Klein SL, Marks MA, Li W, et al. Sex differences in the incidence and case fatality rates from hemorrhagic fever with renal syndrome in China, 20042008. Clin Infect Dis. 2011;52(12):1414-21. https://doi.org/10.1093/cid/cir232.

7. Heyman P, Vaheri A, Lundkvist, et al. Hantavirus infections in Europe: from virus carriers to a major public-health problem[J]. Expert Rev Anti Infect Ther. 2009;7(2):205-17. https://doi.org/10.1586/14787210.7.2.205. 
8. Fan H, Zhao Y, Song FC. Acute pancreatitis associated with hemorrhagic fever with renal syndrome: clinical analysis of 12 cases[]]. Ren Fail. 2013; 35(10):1330-3. https://doi.org/10.3109/0886022X.2013.828187.

9. Park KH, Kang YU, Kang SJ, et al. Short report: experience with Extrarenal manifestations of hemorrhagic fever with renal syndrome in a tertiary Care Hospital in South Korea[J]. Am J Trop Med Hyg. 2011;84(2):229-33. https:// doi.org/10.4269/ajtmh.2011.10-0024.

10. Yu PB, Tian HY, Ma CF, et al. J. Epidemiol Infect 2015. 1984-2012;143(2):405-11.

11. Xiao $D$, Wu K, Tan $X$, et al. The impact of the vaccination program for hemorrhagic fever with renal syndrome in Hu County, China[J]. Vaccine. 2014;32(6):740-5. https://doi.org/10.1016/j.vaccine.2013.11.024.

12. Ma C, Yu P, Nawaz M, et al. Hantaviruses in rodents and humans, Xi'an, PR China[J]. J Gen Virol. 2012;93(Pt_10):2227-36.

13. Bradley EL. A clinically based classification system for acute pancreatitis. Summary of the international symposium on acute pancreatitis, Atlanta, Ga, September 11 through 13, 1992. [J]. Arch Surg. 1993;128(5):586.

14. Wacker DA, Winters ME. Shock[J]. Emerg Med Clin North America. 2014; 32(4):747-58.

15. Cohen AL, Calfee D, Fridkin SK, et al. Recommendations for metrics for multidrug-resistant organisms in healthcare settings: SHEA/HICPAC position paper. Infect Control Hosp Epidemiol. 2008;29(10):901-13. https://doi.org/1 $0.1086 / 591741$.

16. Rosenbaum PR, Rubin DB. Constructing a control group using multivariate matched sampling methods that incorporate the propensity score. Am Stat. 1985;39:33-8.

17. Rosembaum PR. The central role of the propensity scores in observational studies for causal effects[]]. Biometrika. 1983;70(1):41-55. https://doi.org/10.1 093/biomet/70.1.41.

18. Austin PC, Grootendorst P, Anderson GM. A comparison of the ability of different propensity score models to balance measured variables between treated and untreated subjects: a Monte Carlo study[J]. Stat Med. 2007;26(4): 734-53. https://doi.org/10.1002/sim.2580.

19. Austin PC. Some methods of propensity-score matching had superior performance to others: results of an empirical investigation and Monte Carlo simulations[]]. Biom J. 2009;51(1):171-84. https://doi.org/10.1002/bimj.200810488.

20. Hullinghorst RL, Steer A. Pathology of epidemic hemorrhagic fever. Ann Intern Med. 1953;38(1):77-101. https://doi.org/10.7326/0003-4819-38-1-77.

21. Bren AF, Pavlovčič SK, Koselj M, et al. Acute renal failure due to hemorrhagic fever with renal syndrome. Ren Fail. 1996;18(4):635-8. https://doi.org/10.31 09/08860229609047688.

22. Bui-Mansfield LT, Torrington KG, Kim T. Acute pancreatitis in patients with hemorrhagic fever with renal syndrome. Mil Med. 2001;166(2):167-70. https://doi.org/10.1093/milmed/166.2.167.

23. Zhu Y, Chen Y, Zhu Y, et al. A retrospective study of acute pancreatitis in patients with hemorrhagic fever with renal syndrome. BMC Gastroenterol. 2013;13(1):171. https://doi.org/10.1186/1471-230X-13-171.

24. Puca E, Harxhi A, Pipero P, et al. Pancreatitis in patient with hemorrhagic fever with renal syndrome: a five-year experience. J Infect Dev Ctries. 2017; 11(11):900-3. https://doi.org/10.3855/jidc.9567.

25. Lee JS. Clinical features of hemorrhagic fever with renal syndrome in Korea. Kidney Int. 1991;40(Suppl 35):S88-93.

\section{Publisher's Note}

Springer Nature remains neutral with regard to jurisdictional claims in published maps and institutional affiliations.

Ready to submit your research? Choose BMC and benefit from:

- fast, convenient online submission

- thorough peer review by experienced researchers in your field

- rapid publication on acceptance

- support for research data, including large and complex data types

- gold Open Access which fosters wider collaboration and increased citations

- maximum visibility for your research: over $100 \mathrm{M}$ website views per year

At BMC, research is always in progress.

Learn more biomedcentral.com/submissions 